\title{
The age of astronomy-related organizations
}

\author{
A. Heck \\ Observatoire Astronomique, 11 rue de l'Université, F-67000 Strasbourg, France \\ e-mail: heck@astro.u-strasbg.fr
}

Received October 23; accepted November 4, 1998

\begin{abstract}
The age of currently active astronomy-related organizations is investigated from comprehensive and upto-date samples. Results for professional institutions, associations, planetariums, and public observatories are commented, as well as specific distributions for astronomyrelated publishers and software producers.

Some events had a clear impact on the rate of foundation of astronomy-related organizations, such as World War I and II, the beginning of space exploration and the landing of man on the Moon, but not all of them affected in the same way Western Europe and North America. It is still premature to assess the impact of the end of the Cold War. A category such as the software producers would of course not exist nor prosper without the advent of the computer age and the subsequent electronic networking of the planet. Other aspects are discussed in the paper.
\end{abstract}

Key words: sociology of astronomy - astronomical databases: miscellaneous

\section{Introduction}

This paper is the continuation of earlier studies devoted to the geographical distributions of observational activities for astronomy (Heck 1998b) and of astronomy-related organizations (Heck 1998c). Among other trends, these papers have illustrated that the well-known socio-economic effect of self-reinforcement is also taking place in the worldwide astronomy community.

The current contribution investigates the age of the astronomy-related organizations, be they professional, for amateur and grand public, or linked to astronomy through their activities or through the products they manufacture and distribute. To our knowledge, this is the first study of this kind.

As in the previous papers, the data originate from the master files for StarGuides (Heck 1998a) and StarWorlds

Send offprint requests to: A. Heck
(Heck et al. 1994), the latter one being the WWW version of the former one which is a classical directory on paper (for a detailed presentation, refer to the previous studies or to Heck 1997). The files are gathering together all practical data available on associations, societies, scientific committees, agencies, companies, institutions, universities, etc., and more generally organizations involved in astronomy and space sciences.

But many other related types of entries have also been included such as academies, advisory and expert committees, bibliographical services, data and documentation centres, dealers, distributors, funding agencies and organizations, journals, manufacturers, meteorological services, museums, norms and standards offices, planetariums, private consultants, public observatories, publishers, research institutions in related fields, software producers and distributors, and so on. Besides astronomy and related space sciences, other fields are also covered when justified (see the previous papers). All the categories have been appropriately flagged in a way that turned out to be very useful to shape up the samples as needed for the current study.

It is appropriate to remind here that we are dealing with validated and authenticated information (from signed and documented questionnaires), systematically compiled and presented, with a permanent updatingprocess scheme. The expertise built up over now almost a quarter of a century in this exercise, as well as the overall stability of the master files, guarantee an excellent exhaustivity of the entries and an homogeneous coverage of the practical data gathered together.

The files used are certainly the best sources available today for the study at hand. It should be recalled here that, contrary to most on-line resources, StarWorlds is not only WWW-oriented, but includes also all the organizations not yet on the web.

The study is based on the foundation years registered by the organizations in the databases mentioned above. Therefore it should be kept in mind that we are not investigating the foundation pattern of astronomy-related 


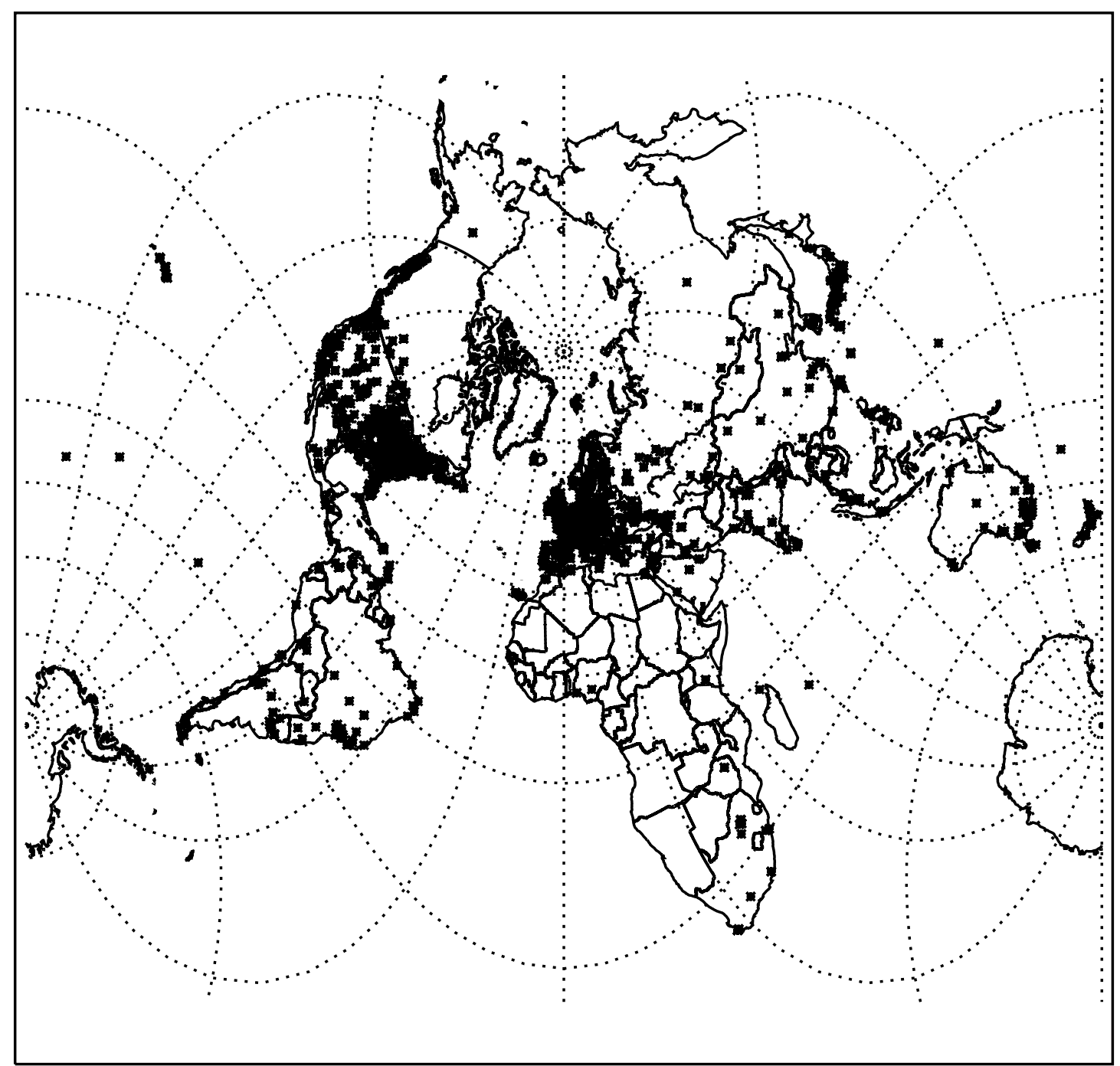

Fig. 1. StarGuides/StarWorlds' organizations with a registered foundation year

organizations over the past centuries, but the age of the organizations still existing and active nowadays.

\section{Foundation years}

A foundation year is explicitly requested on our questionnaires and updating forms. However, when we decided to tackle this study, only $64 \%$ of the organizations had registered one. Therefore we initiated an updating campaign specifically targeted at the database entries without registered foundation year. Additionally and whenever possible, we visited the web sites of the organizations who maintained one and/or issued e-messages requesting explicitly those foundation years. This had a very beneficial impact as the proportion of available foundation years increased to $86 \%$, i.e. 4371 foundation years for a total of 5065 effective database entries (i.e. excluding the crossreferences).
The geographical distributions of the corresponding organizations are illustrated in Figs. 1 to 3 (World, Europe, North America). They are consistent with the distributions in the previous studies. Thus we believe that a study based on such an amount (and such a percentage of the database contents) can lead to quite significant results.

For most organizations (associations, companies, publishers, and so on), the concept of foundation year is an unambiguous one as it corresponds to an official act. For academic institutions, we met some unexpected complications as some of our e-correspondents were looking for the earliest traces of physics/astronomy education in their institutions (as if we had launched a competition for the oldest such teaching in the world). No, our main purpose was to give a faithful picture of the organizational situation as it is today. Therefore the foundation years entered in the database correspond as far as possible to the entities mentioned and not to possible forerunners. Sometimes however, additional information is given when the local 


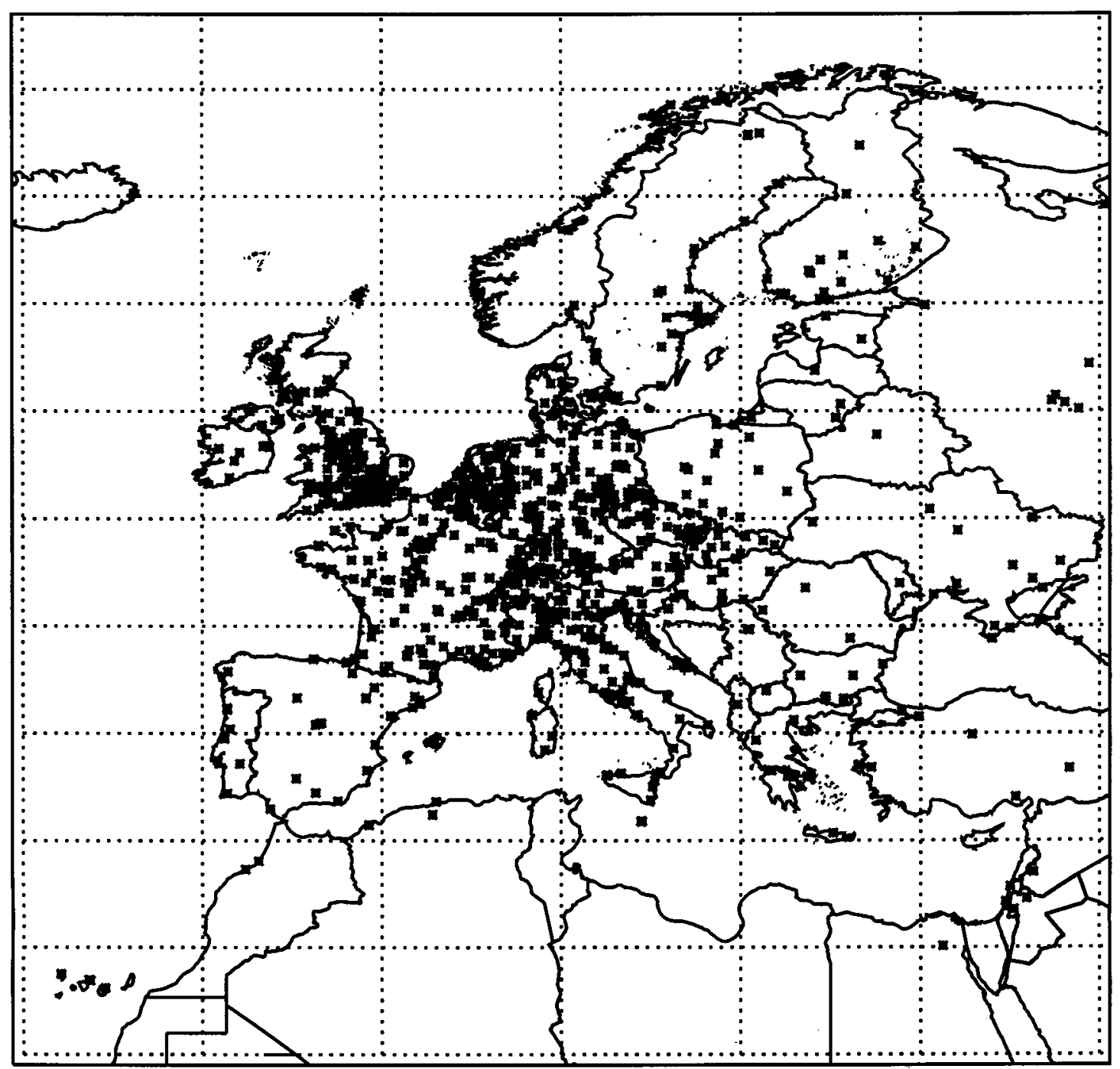

Fig. 2. StarGuides/StarWorlds' organizations with a registered foundation year - European blowup

situation or history justifies it. Finally, it is surprising (but is it really?) how the history of some organizations is poorly known by people working in them.

\section{The samples and distributions}

We have investigated the distributions of foundation years for the categories as described hereafter. The general characteristics are summarized in Table 1 which gives also a weighted average and the corresponding dispersion for each sample.

A specific organization may belong to several categories (typically a public observatory may also host a planetarium and vice versa a planetarium may offer observing facilities to the public). On the other hand, when an organization (typically a commercial company) had several branches at different locations registered with the same foundation year, it counted only for one single occurrence in the statistics. This applied also to large scientific institutions with several entries (divisions, departments, and so on) in the database.

When the worldwide sample was large enough (total sample, institutions, associations), we additionally considered two geographical subsamples: on one hand, a "North American" one made of entries from Canada plus U.S.A. and, on the other hand, a "West European" one consisting basically of all the countries from the European Union, plus Iceland, Norway and Switzerland. As seen from Table 1, such grouping gives samples of similar sizes in two cases out of three (and thus the results of these are directly comparable). There is no special discrimination in leaving out the East European countries (former Socialist Republics): simply those countries have not yet completed their restructuring following the fall of the iron curtain as we experience it daily when updating the databases. Also the obtention of reliable and exhaustive data still remains a problem as of today in some of these countries. 


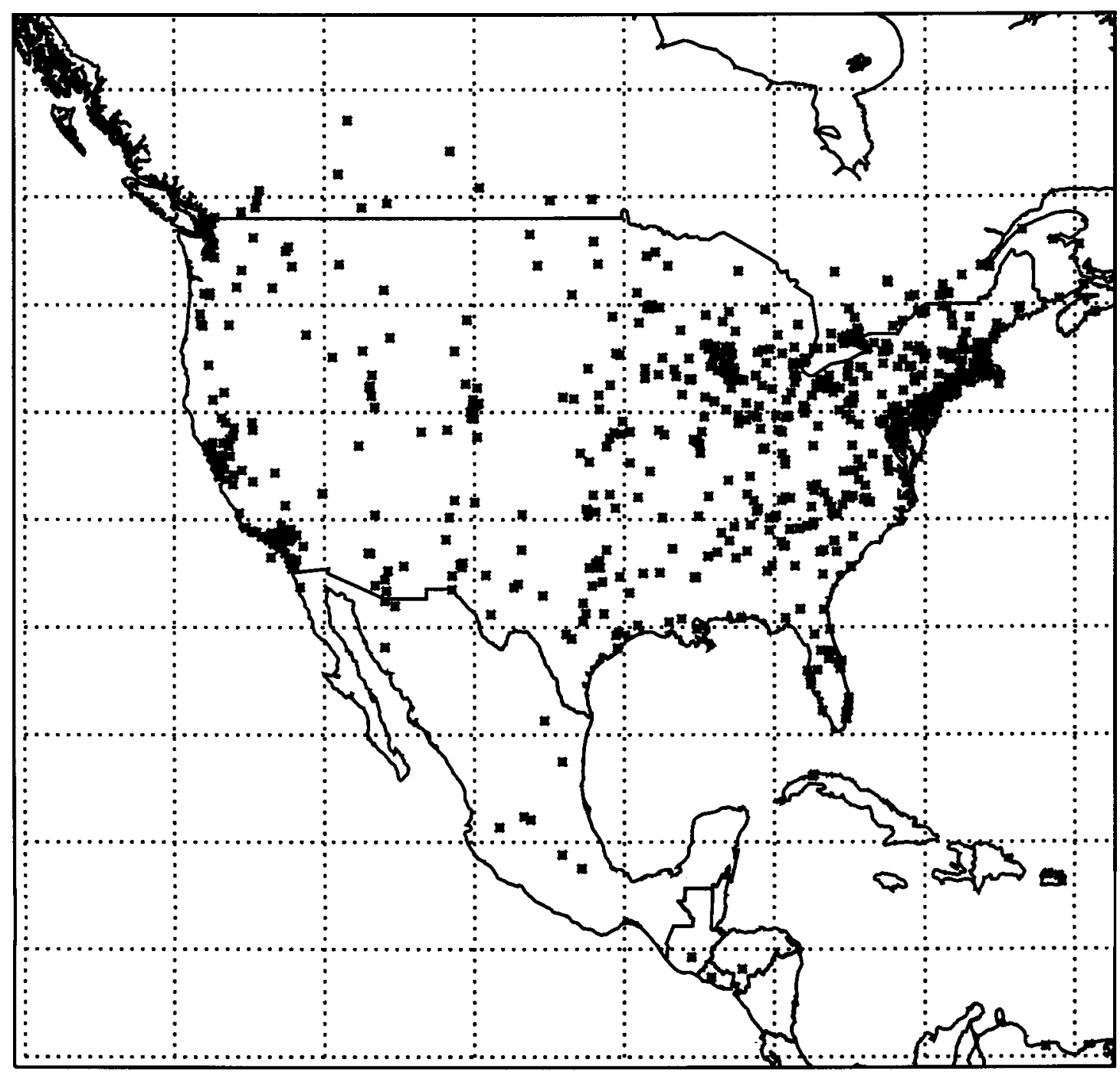

Fig. 3. StarGuides/StarWorlds' organizations with a registered foundation year - North American blowup
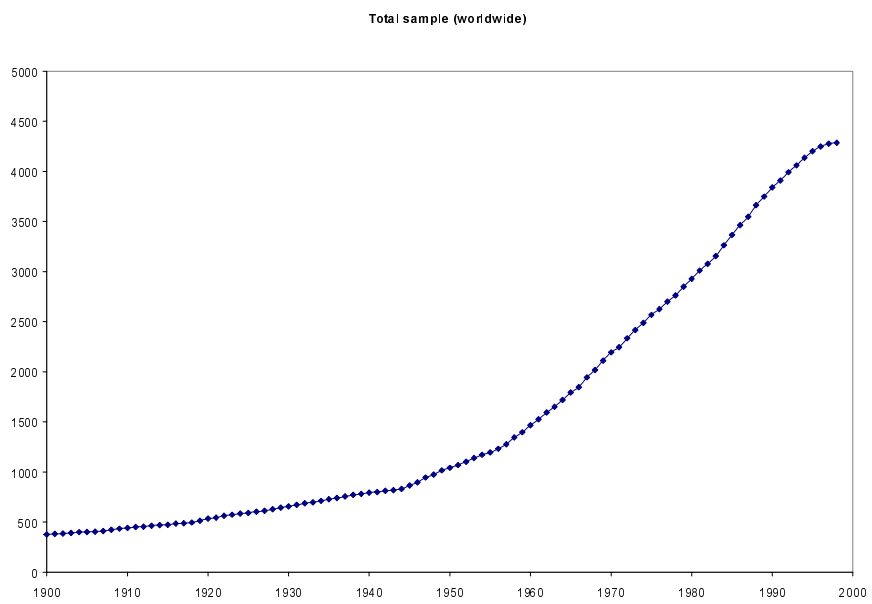

Fig. 4. Cumulative distribution of foundation years for the whole sample (all categories, all countries - see text and Table 1)
Cumulative distribution curves have been used for a better legibility of the significant events. The scale of the abcissa (time/years) has also been chosen for the best legibility of the curves. The ordinate scale has been automatically adapted to the sample size. In any case, additional plots can be requested from the author.

Surges or ruptures of the slope gradient are interesting features as well as plateaux in the otherwise always increasing cumulative curves. A priori, the following periods of time should be perused: World War I (1914-1918), World War II (1939-1945), the launch of Sputnik I (1957) and the landing on the Moon of Apollo 11 (1969), especially for the organizations linked to the public and/or amateurs.

The flattening of the curves towards the end of the ranges is considered as non-significant and attributed to the fact that the most recently created organizations might not be registered yet at the time of writing (Fall 1998). End-of-Cold-War effects are however not excluded. 
Table 1. Samples and properties

\begin{tabular}{|c|c|c|c|c|c|c|c|}
\hline Sample & Description & Size & $\begin{array}{c}\text { Range } \\
\text { start }\left(^{*}\right)\end{array}$ & $\begin{array}{c}\text { Cumulative } \\
\text { total }\end{array}$ & $\begin{array}{c}\text { Weighted } \\
\text { average }\end{array}$ & Sigma & Figure \\
\hline 1 & Total & 4371 & 1279 & $4286\left(^{* *}\right)$ & 1957.6 & 46.3 & 4 \\
\hline 2 & $\begin{array}{c}\text { Total } \\
\text { (Western Europe) }\end{array}$ & 1734 & 1410 & $1704\left(^{* *}\right)$ & 1954.8 & 54.9 & 5 \\
\hline 3 & $\begin{array}{c}\text { Total } \\
\text { (Canada + U.S.A.) }\end{array}$ & 1728 & 1478 & $1701\left(^{* *}\right)$ & 1960.6 & 39.3 & 6 \\
\hline 4 & Institutions & 1066 & 1279 & $979\left(^{* *}\right)$ & 1940.4 & 64.3 & 7 \\
\hline 5 & $\begin{array}{c}\text { Institutions } \\
\text { (Western Europe) }\end{array}$ & 305 & 1410 & $299\left(^{* *}\right)$ & 1930.9 & 81.0 & 8 \\
\hline 6 & $\begin{array}{c}\text { Institutions } \\
\text { (Canada + U.S.A.) }\end{array}$ & 365 & 1746 & $356\left(^{* *}\right)$ & 1943.1 & 51.3 & 9 \\
\hline 7 & Associations & 1019 & 1820 & $1007\left(^{* *}\right)$ & 1970.8 & 23.2 & 10 \\
\hline 8 & $\begin{array}{c}\text { Associations } \\
\text { (Western Europe) }\end{array}$ & 536 & 1820 & 536 & 1972.8 & 22.6 & 11 \\
\hline 9 & $\begin{array}{c}\text { Associations } \\
\text { (Canada + U.S.A.) }\end{array}$ & 342 & 1862 & $331\left(^{* *}\right)$ & 1968.3 & 23.9 & 12 \\
\hline 10 & Planetariums & 416 & 1889 & $408\left(^{* *}\right)$ & 1969.3 & 17.0 & 13 \\
\hline 11 & Public Observatories & 308 & 1675 & 308 & 1964.3 & 33.6 & 14 \\
\hline 12 & Publishers & 144 & 1478 & $127\left(^{* *}\right)$ & 1939.6 & 72.8 & 15 \\
\hline 13 & Software Producers & 157 & 1946 & $154\left(^{* *}\right)$ & 1984.4 & 8.5 & 16 \\
\hline
\end{tabular}

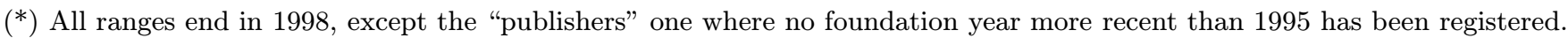
$(* *)$ A value lower than the size of the corresponding sample indicates that some duplications of entries for the same organizations (at different locations, but with the same foundation year) have counted only for one occurrence (see text).

Total sample (Western Europe)

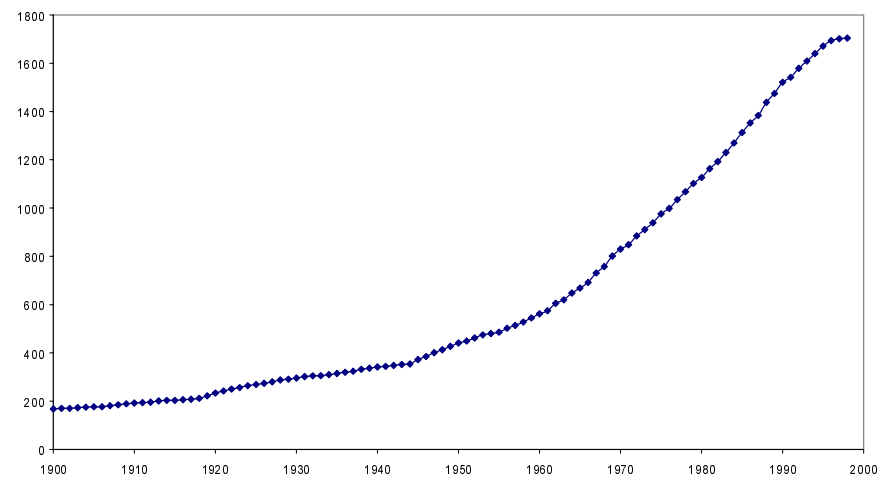

Fig. 5. Cumulative distribution of foundation years for the whole sample (all categories, Western Europe - see text and Table 1)

\subsection{Total sample}

The cumulative distributions for the total sample are illustrated in Figs. 4 to 6 (World, Western Europe, Canada + U.S.A.) from 1900 onwards.

The effect of WWI is noticeable on the West European curve, while the impact of WWII is clearly visible on all curves. A surge at the end of the fifties is striking on the North American curve which is much steeper from then on. The effect is smoother on the West European curve, but the change of steepness is definitely there too. Let us try to identify more precisely those effects in the following subsections.

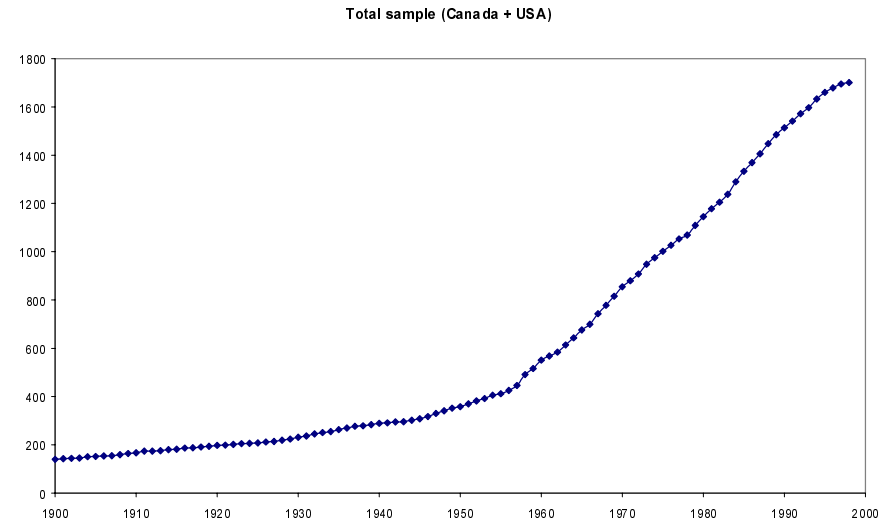

Fig. 6. Cumulative distribution of foundation years for the whole sample (all categories, Canada + U.S.A. - see text and Table 1)

\subsection{Professional institutions}

The cumulative distributions for the academic institutions who clearly indicated an astronomy research/education activity are illustrated in Figs. 7 to 9 (World, Western Europe, Canada + U.S.A.) from 1900 onwards.

Among the oldest institutions, let us mention here: Beijing Astronomical Observatory (1279), Leiden University Observatory (1633), Utrecht University Observatory (1642), Uppsala University Observatory (1650), Paris Observatory (1667), Lund University Observatory (1672), Royal Greenwich Observatory (1675 


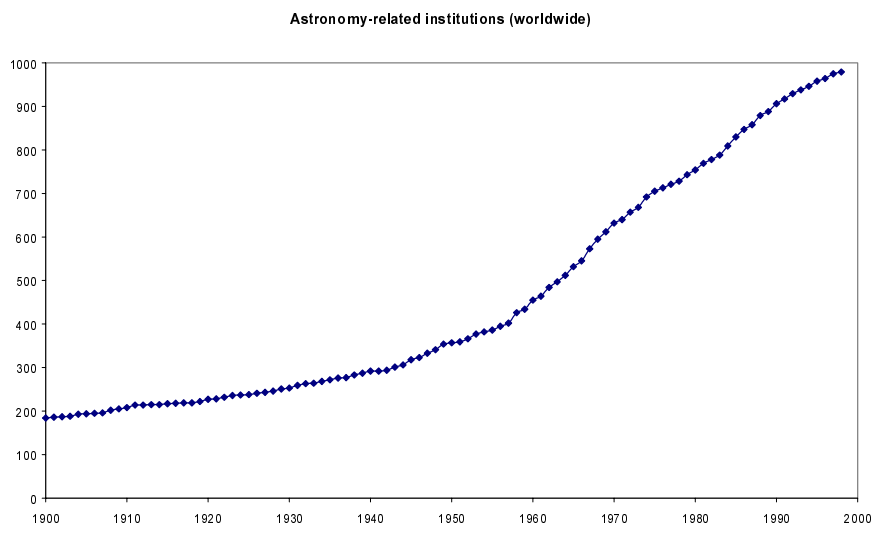

Fig. 7. Cumulative distribution of foundation years for the astronomy-related academic institutions (all countries - see text and Table 1)

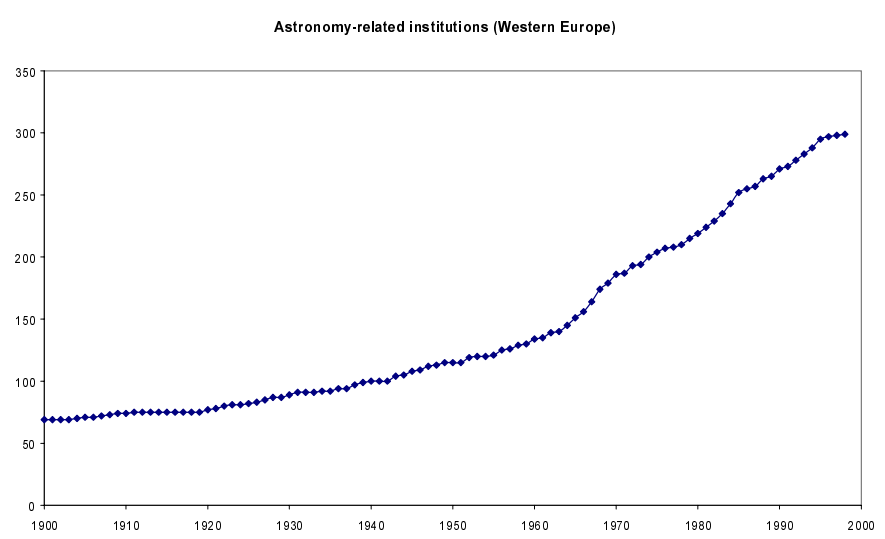

Fig. 8. Cumulative distribution of foundation years for the astronomy-related academic institutions (Western Europe see text and Table 1)

Astronomy related institutions (Canada + USA)

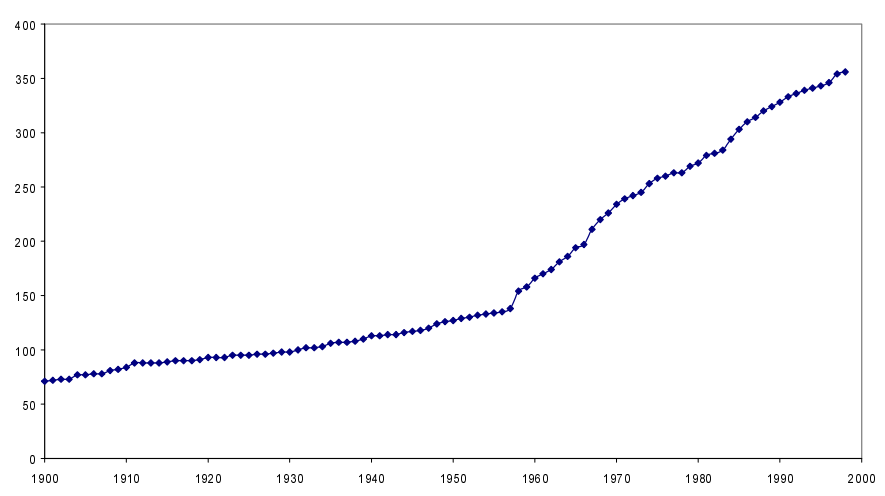

Fig. 9. Cumulative distribution of foundation years for the astronomy-related academic institutions (Canada + U.S.A. see text and Table 1)

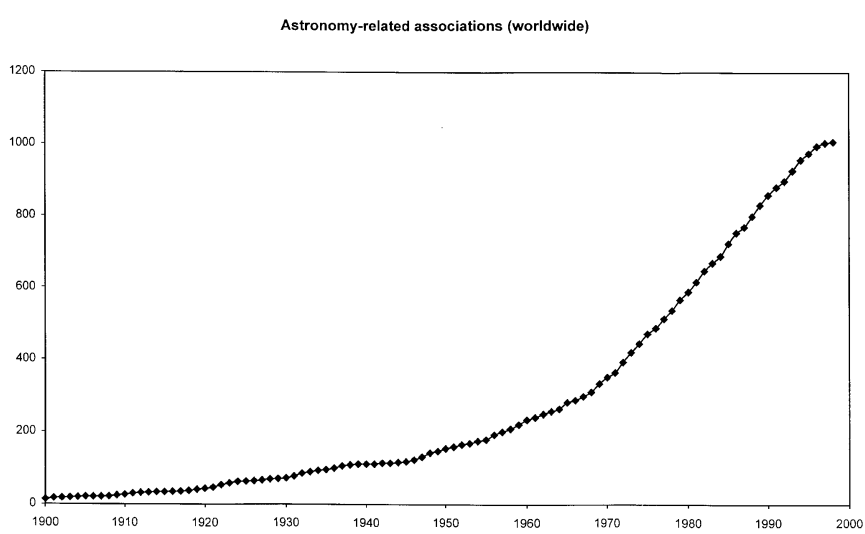

Fig. 10. Cumulative distribution of foundation years for the astronomical associations (all countries - see text and Table 1)

Astronomy-related associations (Western Europe)

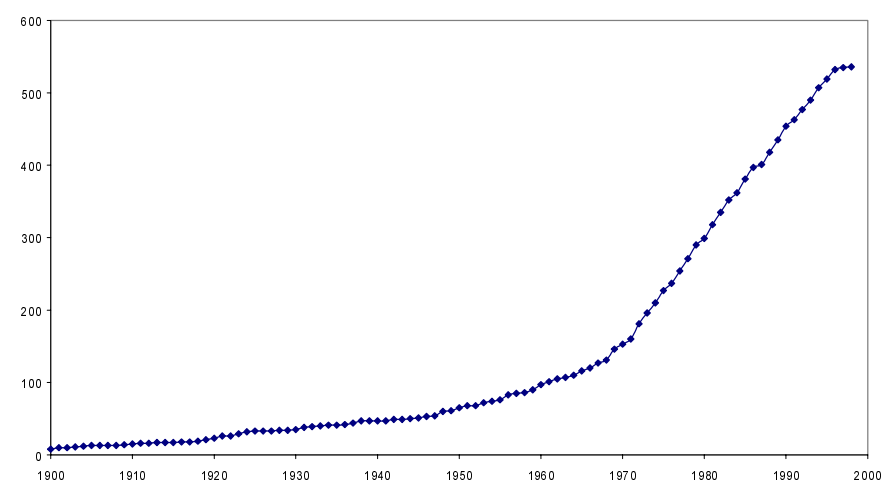

Fig. 11. Cumulative distribution of foundation years for the astronomical associations (Western Europe - see text and Table 1)

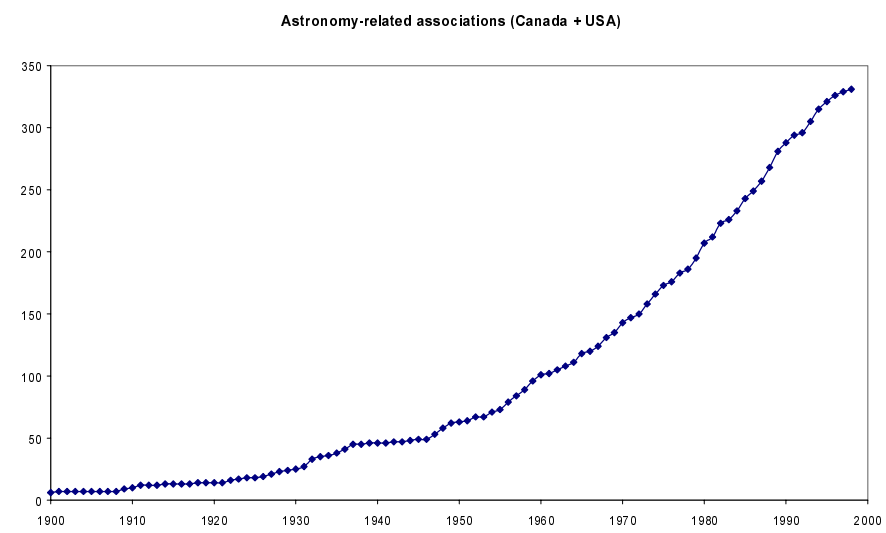

Fig. 12. Cumulative distribution of foundation years for the astronomical associations (Canada + U.S.A. - see text and Table 1) 


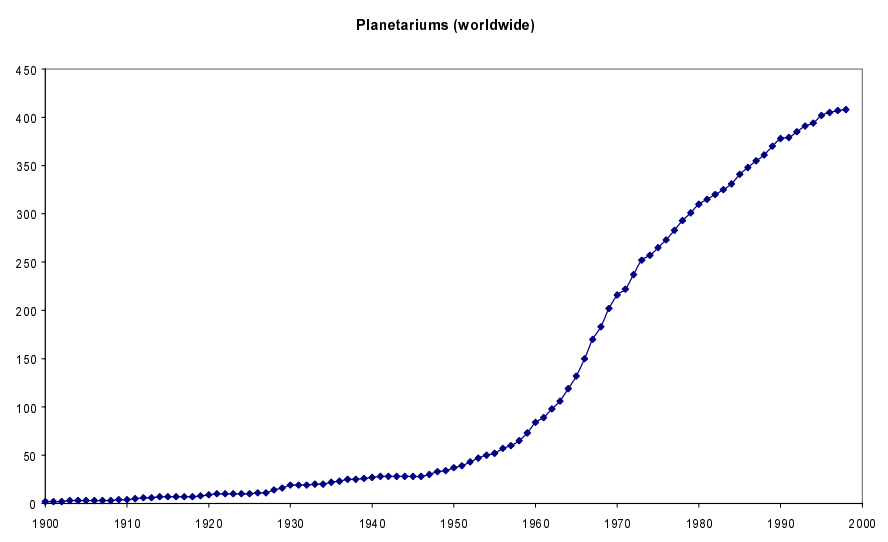

Fig. 13. Cumulative distribution of foundation years for the planetariums (all countries - see text and Table 1)

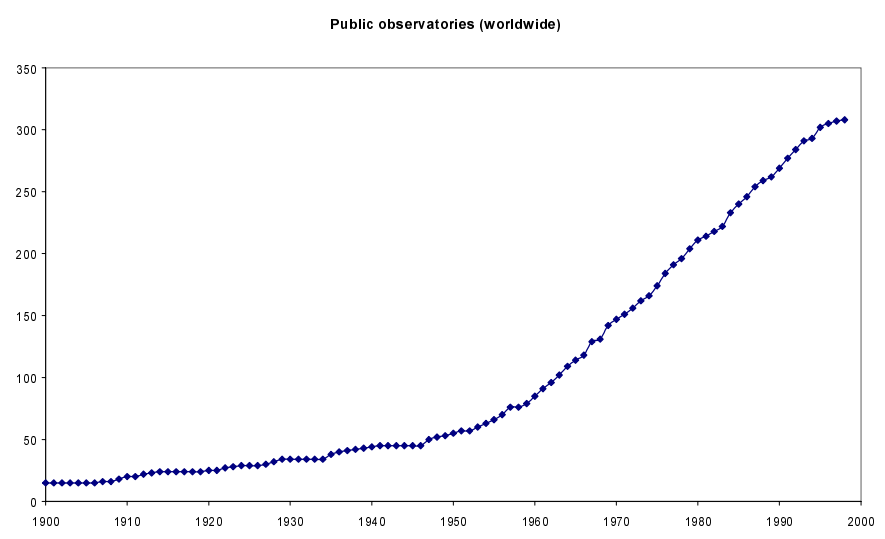

Fig. 14. Cumulative distribution of foundation years for the public observatories (all countries - see text and Table 1)

- but being shut down at the time of writing), and Astronomisches Rechen-Institut (1700).

All the curves display a slow slope increase as time goes with a clear acceleration in the mid-sixties in Europe (the "golden sixties") and at the end of the fifties in North America. In the mid-seventies, the slopes subside until another surge in the mid-eighties.

\subsection{Associations}

The cumulative distributions for the associations who clearly indicated an activity in astronomy is illustrated in Figs. 10 to 12 (World, Western Europe, Canada + U.S.A.) from 1900 onwards.

Here are the major associations founded last century and still active today: Royal Astronomical Society (1820), Chicago Astronomical Society (1862), Astronomische Gesellschaft (1863), Liverpool Astronomical Society (1881), Baltimore Astronomical Society (1881), Société Astronomique de France (1887), Astronomical Society of the Pacific (1889), Royal Astronomical Society of Canada (1890), British Astronomical Association (1890), Astronomical Society of South Africa (1892),

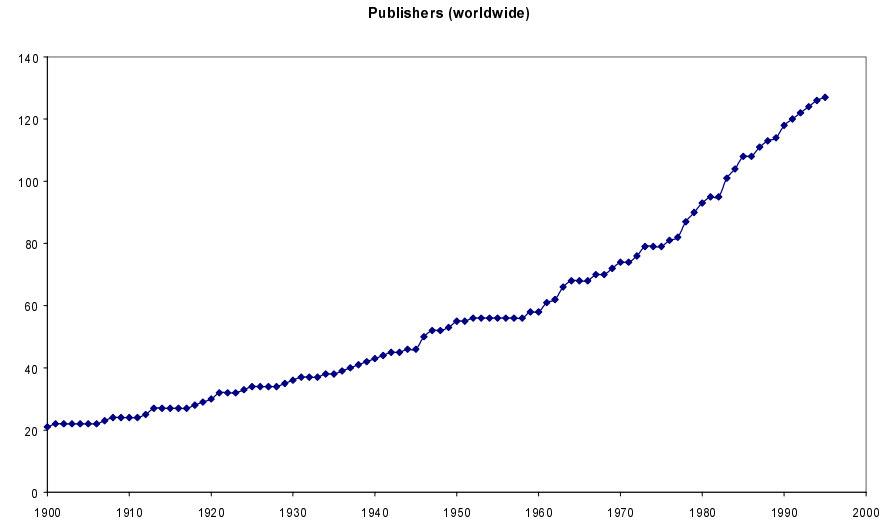

Fig. 15. Cumulative distribution of foundation years for the publishers (all countries - see text and Table 1)

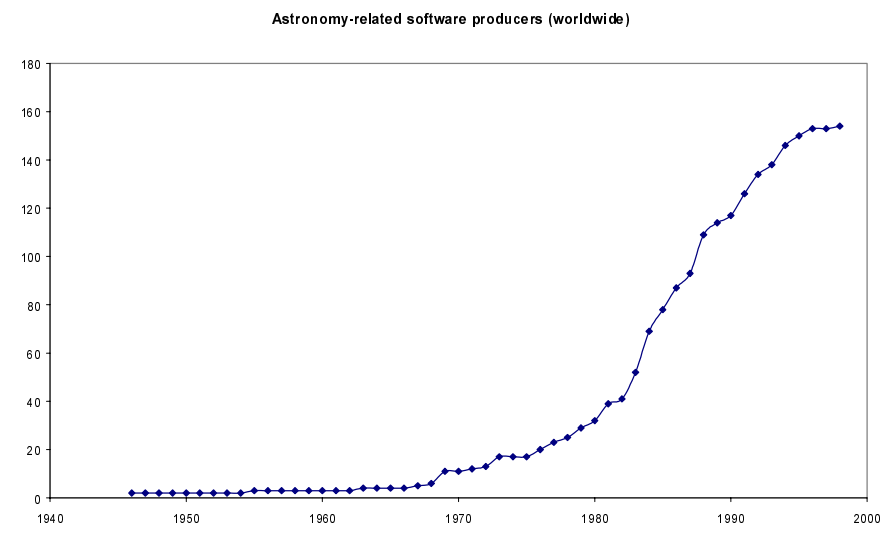

Fig. 16. Cumulative distribution of foundation years for the software producers (all countries - see text and Table 1)

Astronomical Society of Glasgow (1894), and American Astronomical Society (1899).

The curves display slight flattenings at the level of WWI and WWII. The West European curve is significantly smoother than the North American one. Caution however is needed with the different ordinate scales as the sizes of the samples are significantly different.

In other words, from comparable total samples (respectively 1734 and 1728 entries), there are 536 West European associations (31\%), but only 342 North American ones (barely 20\%). This could result from two effects: either the North Americans are less of the associative vein than the West Europeans, or the North American associations have a shorter lifetime. In the latter case, the curve should be much steeper in the recent decades, which does not seem to be case, on the contrary. The weighted averaged foundation years of Table 1 confirm actually that the North American associations are older than the European ones.

As far as surges are concerned, the West European curve shows a few of them, for instance after WWII, around 1970 (Apollo 11 landing on the Moon) and at the mid-eighties. The North American curve has rather the 
surges in the first half of the thirties, after WWII, in the second half of the fifties (Sputnik I) and perhaps at the end of the seventies and of the eighties.

\subsection{Planetariums}

The cumulative distribution of the sample corresponding to the planetariums is illustrated in Fig. 13 from 1900 onwards.

Planetarium activities took off in practice with this century and knew a dramatic increase with the advent of the space exploration from the end of the fifties to the midseventies. The subsequent long-term trend of the curve is subsiding.

It is appropriate to mention here that - as recorded on our questionnaires - a significant number of planetarium facilities are nowadays used also for activities totally unrelated with astronomy.

\subsection{Public observatories}

The cumulative distribution of the sample corresponding to the public observatories is illustrated in Fig. 14 from 1900 onwards.

The oldest public observatory registered in the database is the York Observatory (UK/1831), followed by the Dumfries Observatory (UK/1836), Cincinnati Observatory (U.S.A.-OH/1845) and Sydney Observatory (Australia-NSW/1858).

Slight surges in the curve are noticeable after WWII, at the end of the fifties (Sputnik I) and of the sixties (Man on the Moon), as well as perhaps at the mid-seventies and mid-eighties.

\subsection{Publishers}

The cumulative distribution of the sample corresponding to the astronomy-related publishers is illustrated in Fig. 15 from 1900 onwards. Among the publishing companies founded earlier, one finds the Oxford University Press (1478), Cambridge University Press (1534), Johann Ambrosius Barth (1780), Friedrich Vieweg (1786), Taylor \& Francis (1798), Masson Éditeur (1804), Wiley \& Sons (1807), Springer-Verlag (1842), Hirzel Verlag (1853), and Cornell University Press (1869).

Astronomy-related publishing has been a steady activity. A few surges are however noticeable in the curve: in the first half of the twenties (after the plateau corresponding to WWI), in the second half of the forties (after WWII - notice however that the curve is not flat during the war), as well at the mid-sixties, mid-seventies and mid-eighties.

During WWII, Addison-Wesley was founded (1942), as well as Sky Publishing Corp. (1941), University of South Carolina Press (1944) and Vanderbilt University Press
(1940), all in the US, the mainland of which was not touched by the war.

\subsection{Software producers}

The cumulative distribution of the sample corresponding to the astronomy-related commercial software producers is illustrated in Fig. 16. It calls for a very straightforward interpretation: the curve starts with the computer age (ENIAC's turn-on in 1946); a first surge is contemporaneous with Arpanet's commissioning (1969); after the mid-seventies, the electronic networks started spreading over the world; the eighties saw the popularization of the personal computers; and the first half of the nineties saw the advent of the WWW.

\section{Additional comments}

When dealing with this kind of statistical distributions, it is important to refrain from pushing the analysis of the data too far and to stay at an appropriate level: a global analysis, rather than a case-per-case perusal - and this is especially true for the smaller samples.

If some of the surges or flattenings of the curves are clearly identified (WWI, WWII, beginning of space exploration, man on the Moon), others are less obvious (midseventies? mid-eighties?) and may be due to general economic fluctuations.

Two candid comments are however in order here: in order to issue some appreciation on the general development of astronomy-related activities, the curves displayed here should be compared with similar ones for other disciplines or even more globally for research - unavailable so far to our knowledge; also the rate of creation of astronomyrelated organizations since the end of the fifties is really impressive - and there is no indication that this should still go on that way half a century later, especially at a time when the society at large has other priorities (health, unemployment, security, and so on) than space investigations or cosmological perceptions.

It is still too early to assess the real impact of the end of the Cold War (and of the subsequent reduction of subventions for fundamental research, including astronomy and space sciences). It might already be visible in the flattening of the curves in the nineties, but we believe that no authoritative conclusion is possible right now and that a similar study should be done again with more distance in ten or twenty years.

A category such as the software producers would of course not exist nor prosper without the advent of the computer age and the subsequent electronic networking of the planet.

The West European and the North American curves are mostly similar, but this is not always the case as illustrated in the previous sections. 
Not surprisingly, the second oldest sample on the average is the "publishers" one (and with the second largest dispersion), while the software producers constitute the youngest one (and with the smallest dispersion). The second smallest dispersion corresponds to the "planetariums" sample (as explained above, an activity born roughly with this century), while the largest dispersion is achieved by the West European institutions, making up the oldest sample on the average.

Acknowledgements. We are very grateful to all persons and organizations who contributed over the past quarter of the century to the very substance of the master files used here by returning the questionnaires, by providing the relevant documentation, by participating in the various procedures of maintenance, validation and verification of the information, or otherwise.

\section{References}

Heck A., 1997, Electronic yellow-page services: The Star*s Family as an example of diversified publishing, in Electronic Publishing for Physics and Astronomy, Heck A. (ed.). Kluwer Acad. Publ., Dordrecht, p. 221-220, see also the URL: http://vizier.u-strasbg.fr/starpages.html

Heck A., 1998a, StarGuides 1998 - A directory of astronomy, space sciences and related organizations of the world, Publ. Spéc. CDS 30, viii + 1068 pp., ISBN 2-908064-28-6

Heck A., 1998b, Geographical distribution of observational activities in astronomy, A\&AS 130, 403-406

Heck A., 1998c, Astronomy-related organizations over the world, A\&AS 132, 65-81

Heck A., Egret D., Ochsenbein F., 1994, A\&AS 108, 447-448, see also the URLs: http://vizier.ustrasbg.fr/starworlds.html and http://vizier.u-strasbg.fr/ starbits.html 